\title{
DISCLAIMER
}

This report was prepared as an account of work sponsored by an agency of the United States Government. Neither the United States Government nor any agency thereof, nor any of their employees, makes any warranty, express or implied, or assumes any legal liability or responsibility for the accuracy, completeness, or usefuiness of any information, apparatus, product, or process disclosed, or represents that its use would not infringe privately owned rights. Reference herein to any specific commercial product, process, or service by trade name, trademark, manufacturer, or otherwise does not necessarily constitute or imply its endorsement, recommendation, or favoring by the United States Government or any agency thereof. The views and opinions of authors expressed herein do not necessarily state or reflect those of the United States Government or any agency thereof.

\section{Bayesian Methods for the Combination of Core Sampling Data with Historical Models for Tank Characterization}

\author{
J. C. York \\ K. M. Remund \\ G. Chen \\ B. C. Simpson ${ }^{(a)}$ \\ T. M. Brown ${ }^{(2)}$
}

July 1995

Prepared for

the U.S. Department of Energy

under Contract DE-AC06-76RLO 1830

Pacific Northwest Laboratory

Richland, Washington 99352

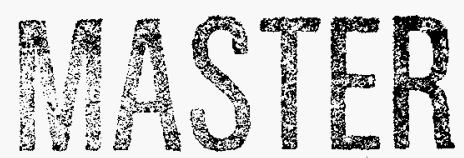

(a) Westinghouse Hanford Company 


\title{
DISCLAIMER
}

This report was prepared as an account of work sponsored by an agency of the United States Govemment. Neither the United States Government nor any agency thereof, nor Battelle Memorial Institute, nor any of their employees, makes any warranty, express or implied, or assumes any legal liability or responsibility for the accuracy, completeness, or usefulness of any information, apparatus, product, or process disclosed, or represents that its use would not infringe privately owned rights. Reference herein to any specific commercial product, process, or service by trade name, trademark, manufacturer, or otherwise does not necessarily constitute or imply its endorsement, recommendation, or favoring by the United States Government or any agency thereof, or Battelle Memorial Institute. The views and opinions of authors expressed herein do not necessarily state or reflect those of the United States Government or any agency thereof.

\author{
PACIFIC NORTHWEST LABORATORY \\ operated by \\ BATTELLE MEMORIAL INSTITUTE \\ for the \\ UNITED STATES DEPARTMENT OF ENERGY \\ under Contract DE-ACO6-76RLO 1830
}

Printed in the United States of America

Available to DOE and DOE contractors from the

Office of Scientific and Technical Information, P.O. Box 62, Oak Ridge, TN 37831; prices available from (615) 576-8401.

Available to the public from the National Technical Information Service, U.S. Department of Commerce, 5285 Port Royal Rd., Springfield, VA 22161 


\section{DISCLAIMER}

Portions of this document may be illegible in electronic image products. Images are produced from the best available original document. 


\begin{abstract}
This document describes a pilot study on the applicability of Bayesian techniques for the combination of historical and analytical data for waste tank characterization. An overview of the analysis and the methods is given, with detailed technical information in several appendixes.
\end{abstract}

Prepared for Westinghouse Hanford Company and the U.S. Department of Energy under Contract DE-AC06.76RLO 1830. 


\section{Acknowledgments}

The authors wish to acknowledge the assistance of Dr. S. F. Agnew of the Los Alamos National Laboratory in preparing this report. 


\section{Contents}

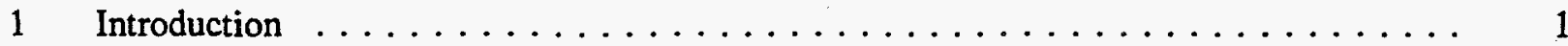

2 Overview of Methodology $\ldots \ldots \ldots \ldots \ldots \ldots \ldots \ldots \ldots \ldots$

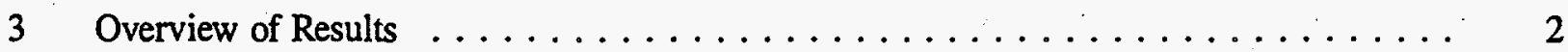

3.1 Analysis of Tanks B-111 and B-201 for Fluoride $\ldots \ldots \ldots \ldots \ldots \ldots \ldots$

3.2 Predictions for $\operatorname{Tank} \mathrm{T}-111 \ldots \ldots \ldots \ldots \ldots \ldots \ldots \ldots \ldots$

4 Conclusions and Recommendations $\ldots \ldots \ldots \ldots \ldots$

A Monte Carlo Analysis of Historical Model $\ldots \ldots \ldots \ldots \ldots \ldots \ldots \ldots$

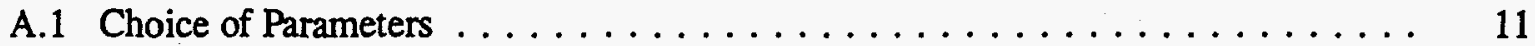

A.2 Specification of Uncertainty about Parameters $\ldots \ldots \ldots \ldots \ldots \ldots$

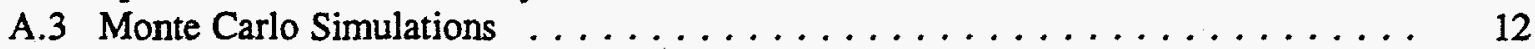

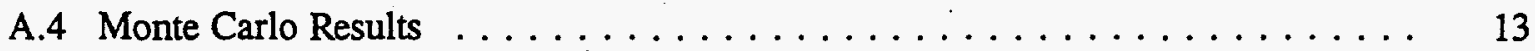

B The Bayesian Analysis $\ldots \ldots \ldots \ldots \ldots \ldots \ldots \ldots \ldots \ldots \ldots$

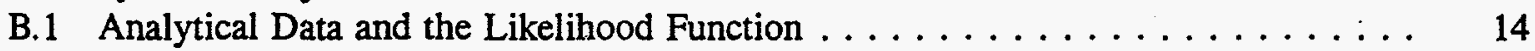

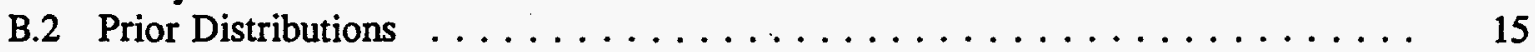

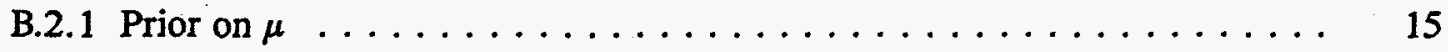

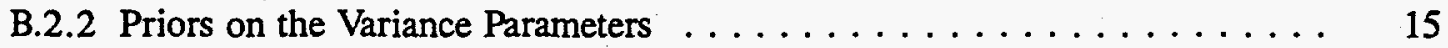

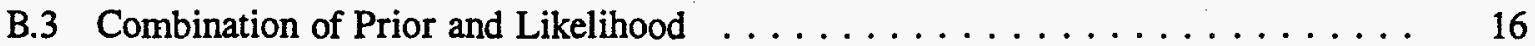

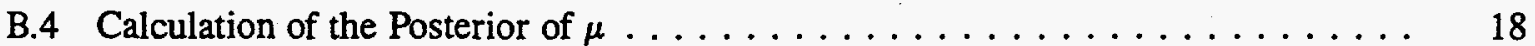

B.5 Updating the Historical Model Parameters $\ldots \ldots \ldots \ldots \ldots \ldots \ldots$

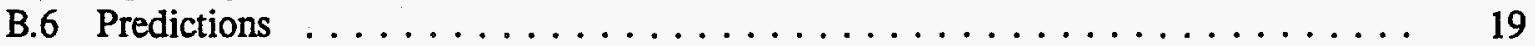

C Parameter Values Used in Prior Distributions $\ldots \ldots \ldots \ldots \ldots \ldots \ldots$ 


\section{Figures}

1 Prior distribution for $\mathrm{B}-111$ fluoride concentration $\ldots \ldots \ldots \ldots$

2 Prior distribution for $\mathrm{B}-201$ fluoride concentration $\ldots \ldots \ldots \ldots \ldots \ldots$

3 Posterior distribution for $\mathrm{B}-111$ fluoride concentration $\ldots \ldots \ldots \ldots$

4 Posterior distribution for B-201 fluoride concentration $\ldots \ldots \ldots \ldots \ldots$

\section{Tables}

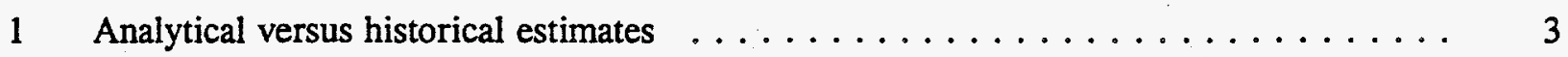

2 Features of the prior and posterior distributions of fluoride concentration for

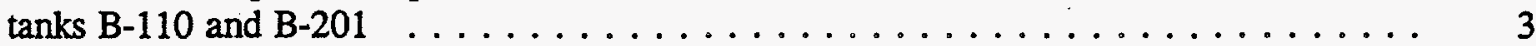

3 Prediction of fluoride concentration for $\mathrm{T}-111 \ldots \ldots \ldots \ldots$

4 Monte Carlo Simulated Concentration Estimates $\ldots \ldots \ldots \ldots \ldots \ldots$

5 Precisions of estimates of laboratory standards $\ldots \ldots \ldots \ldots \ldots \ldots \ldots \ldots$

6 Prior parameters for variance quantities $\ldots \ldots \ldots \ldots \ldots \ldots \ldots \ldots \ldots$ 


\section{Introduction}

A wide variety of information is available on the contents of the nuclear waste tanks at the Hanford site. This report describes an attempt to combine several sources of information using a Bayesian statistical approach. This methodology allows the combination of multiple disparate information sources. After each source of information is summarized in terms of a probability distribution function ( $\mathrm{pdf}$ ), Bayes' theorem is applied to combine them.

This approach has been applied to characterizing tanks B-110, B-111, and B-201. These tanks were chosen for their simple waste matrices: B-110 and B-111 contain mostly $2 \mathrm{C}$ waste, and B-201 contains mostly 224 waste. Additionally, the results of this analysis are used to make predictions for tank T-111 (which contains both $2 \mathrm{C}$ and 224 waste). These predictions are compared to the estimates based on core samples from tank T-111.

\section{Overview of Methodology}

Sections 2 and 3 of this report describe the methodology used, with further detail provided in Appendixes $\mathrm{A}$ and $\mathrm{B}$. The first source of information is the historical tank inventory model developed by Agnew [1]. It incorporates process knowledge, accounting records and other sources of data in order to estimate tank contents. To use this model in the Bayesian analysis, uncertainties must be placed on the estimates that the model produces. This assessment is done with a Monte Carlo simulation study [6].

The other information source is the data on core samples taken from the waste tanks. The statistical models for variability in these core samples provide the probability distributions needed for a Bayesian analysis. This data will be referred to as analytical data.

The Bayesian approach proceeds as follows. Initially, only the historical estimates and their uncertainties are considered; the pdf on these estimates is called the prior distribution, because it summarizes the information available prior to data collection. The function that summarizes the collected data is called the likelihood. Bayes' rule provides the means for combination of the prior and the likelihood to form the posterior distribution, so-called because it summarizes all information available after the data is observed.

This combination of information is not performed blindly, however. It is often the case that the historical model and the core sampling data give very different estimates. In this case, it would be unwise to present a combined estimate, because it is likely that one or both sources of information are subject to biases. In some cases, this will lead to a combined estimate that is representative of neither the analytical nor the historical estimate. In other cases, one or the other estimate will dominate the results. To avoid such problems, we first derive a score which compares the extent to which the two estimates are in agreement. The Bayesian combination of information is performed only when this index indicates a satisfactory agreement between the historical and analytical estimates.

The Bayesian approach can also be used to include the analytical data in the historical model. If one accepts the premise that the analytical data is (for practical purposes) unbiased and gives the best available indication of tank contents, then it is desirable to in- 
corporate the analytical data into the historical model. For the tank(s) for which data has been incorporated, the updated historical model produces estimates and uncertainties that correspond precisely to the posterior distribution. Moreover, for other tanks, the updated model produces estimates based on process knowledge and history, as well as on relevant analytical information. Although this approach has not been implemented, the techniques are described in detail below.

\section{Overview of Results}

A comparison of the Monte Carlo analysis of the historical model with the analytical data showed that, in most cases, it would be inappropriate to combine the two sources of information. For many analytes, one or both of the sources of information is unreliable. This conclusion is arrived at because the discrepancies between the two estimates are much larger than the uncertainties associated with the estimates would indicate. For this reason, the only analyte for which a Bayesian analysis was performed was fluoride.

The analytical and historical estimates of concentrations and their associated uncertainties are reported in Table 1. Additionally, the difference between these two estimates is given, scaled by the square root of the sum of their variances. If indeed the two estimates are centered at the same value, this scaled difference is a quantity which should have mean 0 and variance 1. It can be interpreted in a similar manner to a $\mathrm{Z}$ or $\mathrm{t}$ statistic; however, no $p$-values are reported. This value is an index of agreement between the estimates rather than a formal test statistic: Note that a positive sign means that the historical estimate is larger than the analytical estimate.

Because one goal of this study was to attempt to combine the information learned from tanks B-110 and B-111 with information from tank B-201 in order to predict tank T-111, we required all of the deviations for an analyte to be less than 3 in absolute value. Also, for those analytes for which the historical model predicts a zero concentration, no useful uncertainty can be attached to that estimate. For this reason, lanthanum and manganese were not analyzed further. Fluoride is the only analyte that satisfies all these criteria.

\subsection{Analysis of Tanks B-111 and B-201 for Fluoride}

Following the procedures described in the appendixes, a Bayesian analysis was conducted for fluoride concentrations in B-111 and B-201. For technical reasons described in Appendix B, only one tank of each waste type was analyzed. Although simultaneous analysis of multiple tanks (e.g. B-110 and B-111) is certainly possible, it is not done here for the sake of simplicity.

Figures 1 and 2 show the prior distributions for the fluoride concentrations in tanks B-111 and B-201 respectively. Figures 3 and 4 show the posterior distributions for the two tanks. Table 2 shows summaries of the posterior distributions.

In both tanks, the analytical data has a much larger impact upon the posterior distribution than does the prior. This is because the uncertainty in the analytical data is small relative to the uncertainty in the historical estimate. 


\begin{tabular}{|c|c||r|r||r|r||r|}
\hline Analyte & Tank & Analytic & \multicolumn{1}{|c|}{ sd } & Historical & \multicolumn{1}{c||}{ sd } & Deviation \\
\hline \hline \multirow{3}{*}{$\mathrm{Mn}$} & $\mathrm{B}-110$ & 66.8 & 6.7 & 0.0 & 0.0 & -10.0 \\
\cline { 2 - 7 } & $\mathrm{B}-111$ & 111.0 & 2.2 & 0.0 & 0.0 & -50.0 \\
\cline { 2 - 7 } & $\mathrm{B}-201$ & 19200.0 & 5568.0 & 150.8 & 19.8 & -3.4 \\
\hline \hline \multirow{3}{*}{$\mathrm{P0}_{4}$} & $\mathrm{~B}-110$ & 25250.0 & 757.5 & 73223.3 & 39409.6 & 1.2 \\
\cline { 2 - 7 } & $\mathrm{B}-111$ & 23900.0 & 717.0 & 68027.9 & 36864.6 & 1.2 \\
\cline { 2 - 7 } & $\mathrm{B}-201$ & 1210.0 & 181.5 & 10948.1 & 1326.2 & 7.3 \\
\hline \hline $\mathrm{F}$ & $\mathrm{B}-110$ & 1895.0 & 113.7 & 13597.4 & 13600.9 & 0.9 \\
\cline { 2 - 7 } & $\mathrm{B}-111$ & 1560.0 & 31.2 & 12614.5 & 12630.9 & 0.9 \\
\cline { 2 - 7 } & $\mathrm{B}-201$ & 5830.0 & 116.6 & 38413.8 & 33614.5 & 1.0 \\
\hline \hline $\mathrm{Fe}$ & $\mathrm{B}-110$ & 18060.0 & 722.4 & 40821.1 & 6405.7 & 3.5 \\
\cline { 2 - 7 } & $\mathrm{B}-111$ & 17700.0 & 885.0 & 46676.6 & 5935.1 & 4.8 \\
\cline { 2 - 7 } & $\mathrm{B}-201$ & 13400.0 & 2680.0 & 40069.4 & 6879.8 & 3.6 \\
\hline \hline \multirow{3}{*}{$\mathrm{Bi}$} & $\mathrm{B}-110$ & 18520.0 & 1111.2 & 20426.1 & 3287.1 & 0.5 \\
\cline { 2 - 7 } & $\mathrm{B}-111$ & 20200.0 & 202.0 & 18929.4 & 3051.6 & -0.4 \\
\cline { 2 - 6 } & $\mathrm{B}-201$ & 94500.0 & 2835.0 & 18730.6 & 3177.4 & -17.8 \\
\hline \hline \multirow{2}{*}{$\mathrm{La}$} & $\mathrm{B}-110$ & 31.81 & 9.2 & 0.0 & 0.0 & -3.4 \\
\cline { 2 - 6 } & $\mathrm{B}-111$ & 11.30 & 3.0 & 0.0 & 0.0 & -3.7 \\
\cline { 2 - 7 } & $\mathrm{B}-201$ & 15100.0 & 1510.0 & 24148.8 & 4033.1 & 2.101 \\
\hline \hline
\end{tabular}

Table 1: Analytical versus historical estimates. Note that the historical model assumes no $\mathrm{La}$ or $\mathrm{Mn}$ is present in tanks B-110 and B-111. The historical values reported are the means of the prior distributions generated via the Monte Carlo analysis, and so may not agree with published historical estimates. Any discrepancies arise because the values in this table represent average outputs given a range of inputs to the historical model, whereas the published historical results give the one output corresponding to one particular input.

\begin{tabular}{|c|c|c|c|c|c|c|c|}
\hline \multirow[b]{2}{*}{ Tank } & \multicolumn{3}{|c|}{$\overline{\text { Prior }}$} & \multicolumn{3}{|c|}{ Posterior } & Analytical \\
\hline & 5th \%ile & Mean & 95th \%ile & 5th \%ile & Mean & 95th \%ile & Estimate \\
\hline B-111 & 1288.1 & 2320.8 & 2404.7 & 1480.2 & 1554.5 & 1631.8 & $1550: 0$ \\
\hline B-201 & 3027.0 & 4133.7 & 10603.6 & 5864.9 & 6011.9 & 6171.2 & 6000.0 \\
\hline
\end{tabular}

Table 2: Features of the prior and posterior distributions of fluoride concentration for tanks B-110 and B-201. 


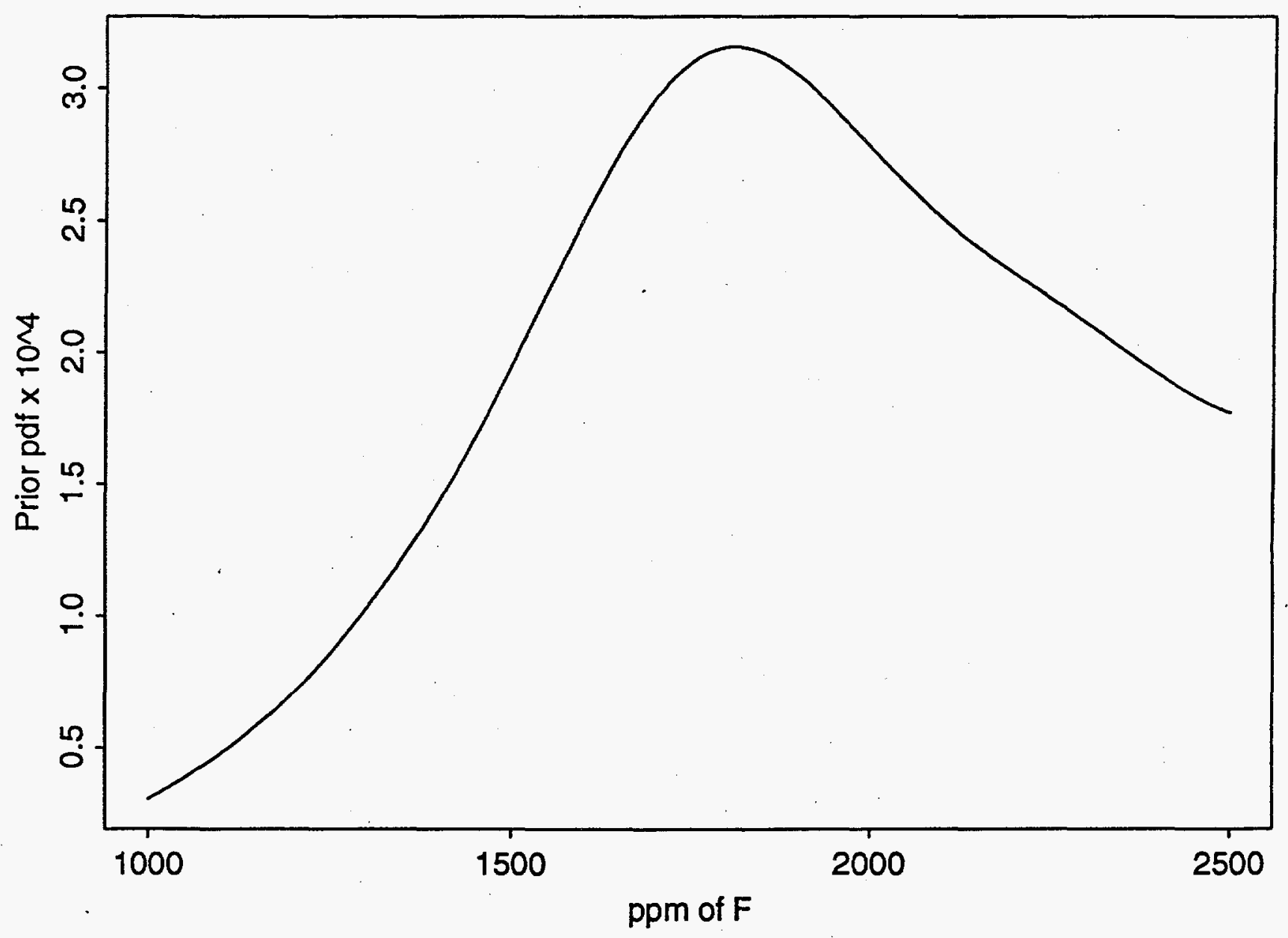

Figure 1: Prior distribution for B-111 fluoride concentration. 


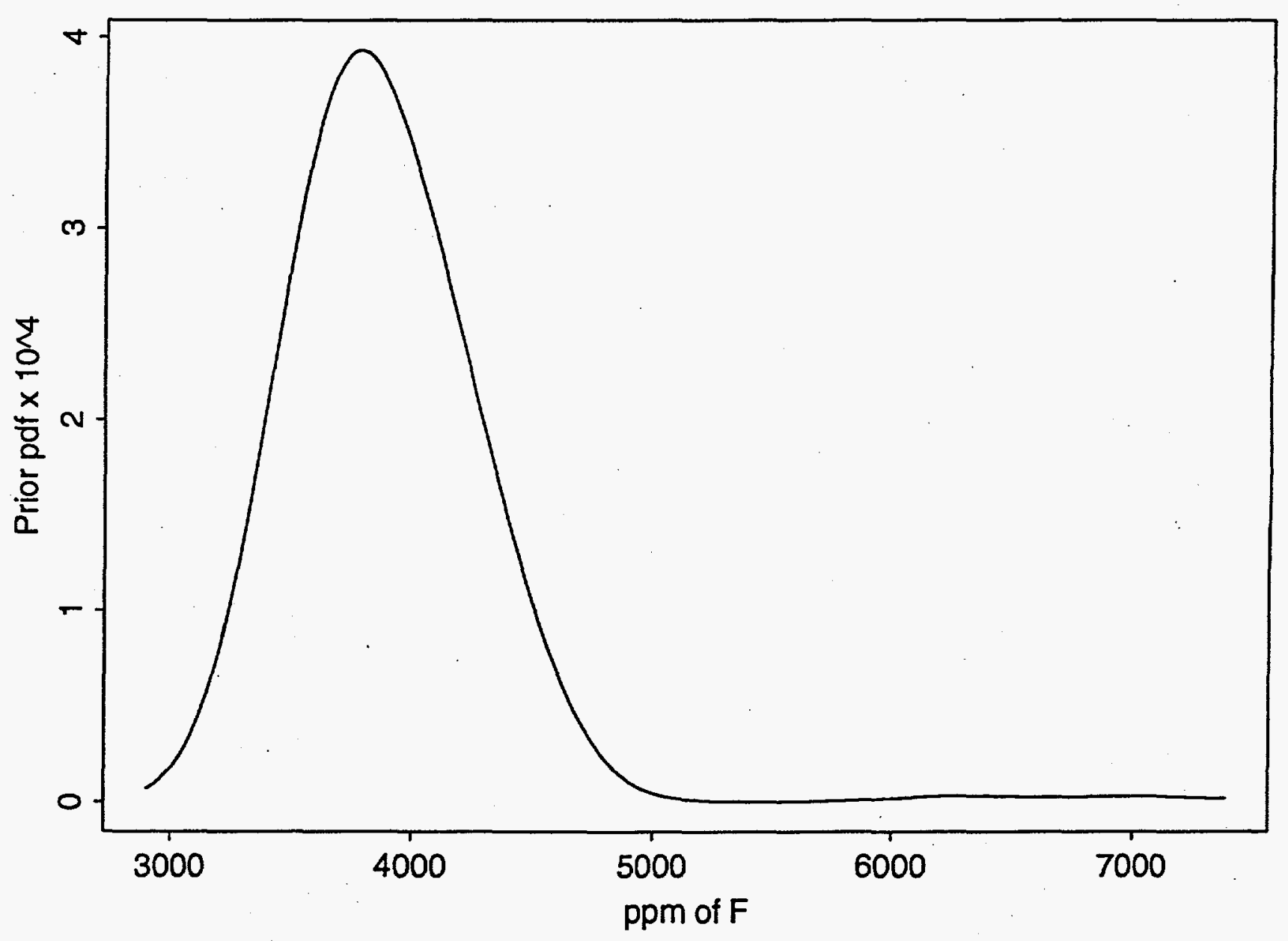

Figure 2: Prior distribution for B-201 fluoride concentration. 


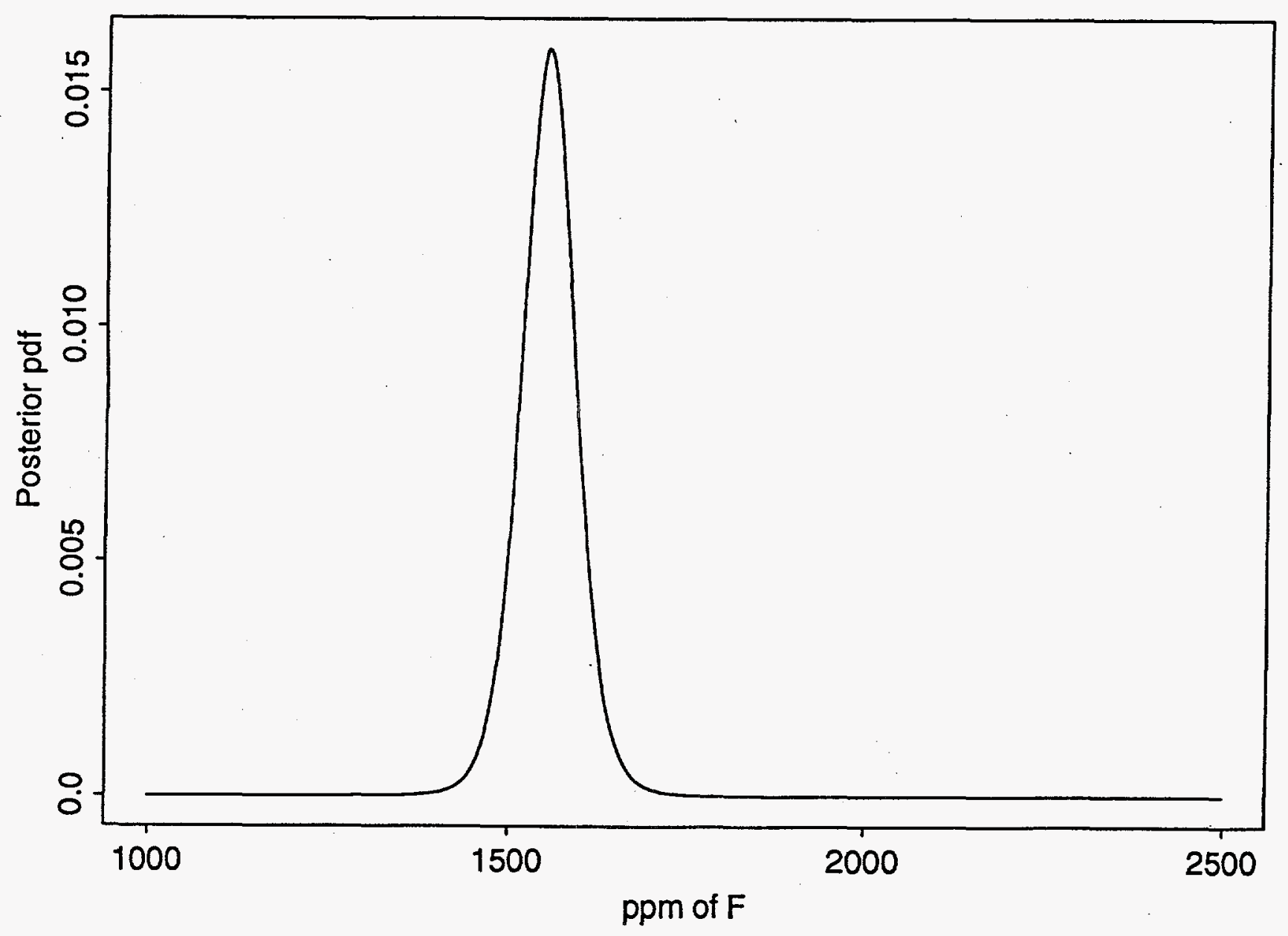

Figure 3: Posterior distribution for B-111 fluoride concentration. 


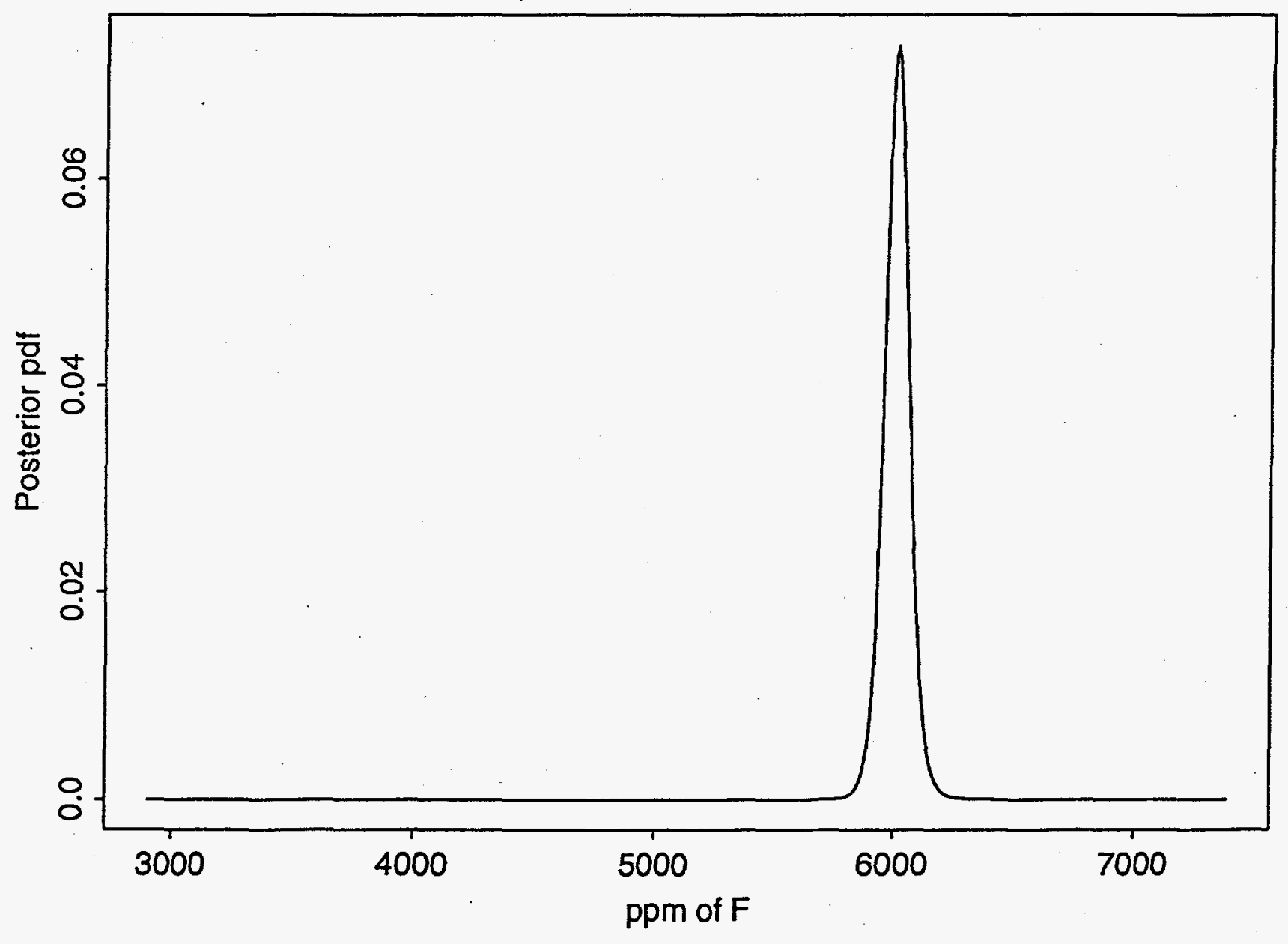

Figure 4: Posterior distribution for B-201 fluoride concentration. 


\subsection{Predictions for Tank T-111}

Based on the analysis for tanks B-111 and B-201, predictions were made for tank T-111. This tank contains a mixture of $2 \mathrm{C}$ and 224 waste, and it was hoped that the knowledge gleaned from the analyses of the single-waste tanks would provide an improved prediction for tank T-111. If the posterior mean is used as a prediction of concentration, the estimate for tank T-111 is a weighted average of the estimates for tanks B-111 and B-201. Tank T-111 is composed of $92 \% 2-\mathrm{C}$ waste and $8 \% 224$ waste, and these proportions are used to weight tanks B-111 and B-201 respectively. As a reminder, this prediction was made without using the analytical data available for T-111, in order to test the accuracy of the approach. The Bayesian estimate is closer to the analytical estimate for tank T-111 than to the original historical estimate (taken from [3]). However, it is difficult to draw any conclusions from only one example. A summary of the predictions is given in Table 3 .

\begin{tabular}{|c|c||c||c|}
\hline $\begin{array}{c}\text { Bayesian } \\
\text { Estimate }\end{array}$ & $\begin{array}{c}\text { RSD of } \\
\text { Estimate }\end{array}$ & $\begin{array}{c}\text { Analytic } \\
\text { Estimate }\end{array}$ & $\begin{array}{c}\text { Historical } \\
\text { Estimate }\end{array}$ \\
\hline 1906.4 & $2.1 \%$ & 2300 & 7130 \\
\hline
\end{tabular}

Table 3: Prediction of fluoride concentration for T-111.

\section{Conclusions and Recommendations}

We have described a feasible and functional mathematical framework for combining the historical model with analytical data for tank characterizations. Ultimately, this approach could be used to make predictions for unsampled tanks, analyze data as it becomes available, fold the knowledge gained back into the historical model, and make improved predictions. However, several issues must be dealt with before implementing such an approach.

1. Improved Monte Carlo Analysis. In our approach, we considered only a small number of the parameters that are input into the historical model. Current and future work will expand the numbers and types of parameters studied.

2. Improved Historical Model. It should be clear from the preceding analyses that discrepancies between the historical and analytical estimates are often too great to justify combining them. The historical model is steadily evolving and improving, via qualitative and quantitative changes based on performance assessments. When it is to a point where it reliably produces estimates which are not incompatible with what analytic information indicates, the approaches described in this document could be applied in a broader and more automatic fashion. This line of reasoning assumes the analytical data is not itself too unreliable or misleading. 
3. Generalized Computational Approach This paper describes a first attempt at producing a framework for analysis, so there were some computational and mathematical issues that could not be investigated in depth. Future work would require extension of the approach described here in two main areas. The likelihoods used, and especially the formula for integrating out the variances, are for a balanced design. For analytical data with more core samples and/or segment-level information, the likelihoods and integrations need to be derived. Also, the more general issue of how to simultaneously analyze several analytes from several tanks needs more consideration.

If the only goal of this work were to provide an estimate of the mean concentration for tanks that had been sampled, the Bayesian approach would not offer any great advantages. In comparing Figure 1 to Figure 3, and 2 to 4 , one can see that the historically based prior had little impact on the posterior. However, one of the primary motivations for this effort was to develop an approach that would allow the historical model to be quantitatively updated and fine-tuned, based upon the analytical data. We have demonstrated that the Bayesian approach is capable of doing this by making the predictions for tank T-111. However, for most analytes it was not advisable to perform this updating. The reasons for the deviations between the historical and analytical estimates are perhaps more qualitative (i.e., the historical model itself needs to be revised) than quantitative (i.e., the parameter values used in the historical model need to be revised). If this is indeed the case, it is better to postpone the Bayesian analysis described here until the qualitative performance of the historical model is improved. At that point, the methods described here provide a powerful way of fine-tuning the historical model, so that predictions for unsampled tanks can be made based on available analytic data, as well as historical information.

In conclusion, we feel that this approach is a formal, mathematically defensible way of providing a single estimate based on multiple sources of data. However, it would not be prudent to go down this path until the reasons for the observed discrepancies between the historical and analytical estimates are understood and dealt with.

\section{References}

[1] Agnew, S.F. "Hanford Defined Wastes: Chemical and Radionuclide Compositions," LAUR-94-2657, 1994.

[2] Agnew, S.F. and Watkin, J.G. "Estimation of Limiting Solubilities for Ionic Species in Hanford Waste Tank Supernates," LAUR-94-3590, 1994.

[3] Brevick, C.H., Gaddis, L.A. and Pickett, W.W. "Historical Tank Content Estimates for Northwest Quadrant," WHC-SD-WM-ER=351, Rev. 0, 1994.

[4] Box, G.E.P. and Tiao, G.C. Bayesian Inference in Statistical Analysis. New York: John Wiley and Sons, 1992. 
[5] Simpson, B.C. "Tank Characterization Report for Tank 241-T111," WHC-EP-0806, 1994.

[6] York, J.C., Remund, K.M., Chen, G., Simpson B.C., Brown T.M. "Discussion of Results of a Monte Carlo Analysis of Historical Tank Inventory Models," letter report to WHC, 1995. 


\section{A Monte Carlo Analysis of Historical Model}

Before the Bayesian analysis, the first step is to specify the prior distributions on the concentrations of various analytes in the waste tank. We take the approach of first identifying inputs (parameters) of the historical model which have a substantial impact upon the predictions, but which cannot be accurately determined from the historical information. We place pdfs on those quantities, and determine the pdf that are thus induced on the model estimates.

The Monte Carlo approach described here makes it possible for us to go from pdf on the inputs to pdf on the concentration estimates derived from the model. We sample parameter values from the pdf placed on them, process them through the model, and record the resulting estimates. Repeating this process numerous times produces a sample of values from the pdf induced upon the concentration estimates.

For simplicity, we have narrowed our attention to the following selection of analytes: La, $\mathrm{Mn}, \mathrm{Bi}, \mathrm{PO}_{4}, \mathrm{SiO}_{3}, \mathrm{Fe}, \mathrm{F}$.

\section{A.1 Choice of Parameters}

Many parameters feed into the historical model. Rather than work with them all, our approach is to choose a small set of them for use in the uncertainty analysis. We chose this set of parameters according to the following criteria:

1. The estimates produced by the model are sensitive to the value of the parameter.

2. The value of the parameter is somewhat uncertain.

The parameters thus selected are the volume percent solids (percentage of the volume of the original waste that was composed of solids) and, for each analyte, the limiting solubility of that analyte. Both of these parameters have an impact on the proportion of an analyte that will be in solid form or dissolved in the interstitial liquid.

Other parameters were ignored, but could be included in future analyses. For each analyte, the concentration of that analyte in the original waste stream is quite important. Another quantity is the volume of each waste type added to the tank. We have been able to ignore this quantity by focusing on single-waste tanks; however, this may be a very important factor in future analyses.

\section{A.2 Specification of Uncertainty about Parameters}

After discussions with Simpson and Brown, it was determined that the volume percent solids parameter for the $2 \mathrm{C}$ waste should have the following characteristics:

1. A Typical value is $6.8 \%$ (this is the value used by Agnew).

2. The value is certainly no lower than $2.8 \%$. 
3. The value is very unlikely to be larger than $8.8 \%$.

4. The value is certainly no higher than $13.5 \%$.

To mimic this characterization, a beta distribution was placed on the parameter, so that it always fell within the bounds $(2.8,13.5)$, had 6.8 as its mean, and 8.8 as its 95 th percentile.

The volume percent solids is not as well known for 224 waste. Agnew addresses this in [1]. Because of this, we used a more diffuse distribution on this quantity. It was assumed to range between 2.8 and 13.5, with a typical value of 3.9 (this is the value used by Agnew). Again, a beta distribution was chosen.

The limiting solubility for each analyte was chosen based on data obtained by Agnew and Watkin [2]. He first gathered data from any available analysis that had been performed on supernatant from tanks. To get the limiting solubility for the model, Agnew dropped the $75 \%$ of the data with the smallest values, and used the mean of the remaining data for the limiting solubility.

We formed our priors on the solubilities by first placing beta distributions on all the data obtained by Agnew [2], and then truncating that distribution so the values it produced were guaranteed to be at least as large as the 75th percentile of the observed data. Appendix $\mathrm{C}$ specifies the details of the prior distributions.

\section{A.3 Monte Carlo Simulations}

Usually, if we know the distribution of two random variables, and we wish to find the distribution of some other variable which is a function of the first two, we can find it through some fairly routine mathematics. In the current context, however, there are two difficulties. The function relating the inputs (volume percent solids, limiting solubility) to the estimated concentration of an analyte is complex. The form of the function will vary depending on the analyte considered.

An alternate approach is to simulate a random value for the volume percent solids and the limiting solubility for each analyte, and then calculate the estimated concentration for each analyte based upon those values. This produces one simulated value for the estimated concentration for each analyte. By repeating this process many times, we produce many simulated values for the concentration of each analyte. Based on this sample, we can estimate the actual distribution of the concentration, per analyte.

In practice, we simulate 1000 values for the input parameters using the distributions described in the previous section, run them through the historical model, and produce 1000 simulated values for the concentration of each analyte. We also perform checks on each simulation, to ensure that the input parameters have not resulted in physical impossibilities, such as a very large void fraction ${ }^{1}$. Any such observations are deleted.

\footnotetext{
${ }^{1}$ The void fraction is one of the historical model outputs, and is based upon the amount of precipitate and the estimated volume of solid waste.
} 


\section{A.4 Monte Carlo Results}

Before performing the full Monte Carlo analysis, we first explored the sensitivity of the estimates to the various input parameters. We found the estimated concentrations of both $\mathrm{PO}_{4}$ and $\mathrm{F}$ were sensitive to both the volume percent solids and the solubility; whereas the rest of the analytes depend primarily on the volume percent solids. For all analytes, both parameters were varied in the Monte Carlo study.

The following table summarizes the results of the uncertainty analysis; the same Monte Carlo runs were used for construction of the priors and the subsequent analyses.

\begin{tabular}{|r|r|r|r|r|}
\hline Analyte/Waste Type & 25th \%ile & Median & Mean & 75th \%ile \\
\hline $\mathrm{La} / 224$ & 0.2128 & 0.256 & 0.2481 & 0.2898 \\
\hline $\mathrm{Mn} / 224$ & 0.003669 & 0.003921 & 0.003859 & 0.004051 \\
\hline $\mathrm{Bi} / 224$ & 0.1095 & 0.1321 & 0.1279 & 0.1497 \\
\hline $\mathrm{Bi} / 2 \mathrm{C}$ & 0.1132 & 0.1267 & 0.1303 & 0.1444 \\
\hline $\mathrm{P0}_{4} / 224$ & 0.1456 & 0.1669 & 0.164 & 0.1846 \\
\hline $\mathrm{P0}_{4} / 2 \mathrm{C}$ & 0.3478 & 1.078 & 1.064 & 1.582 \\
\hline $\mathrm{SiO}_{3} / 2 \mathrm{C}$ & 0.2434 & 0.2705 & 0.2776 & 0.3058 \\
\hline $\mathrm{Fe} / 224$ & 0.8749 & 1.057 & 1.024 & 1.2 \\
\hline $\mathrm{Fe} / 2 \mathrm{C}$ & 0.8168 & 0.9154 & 0.9417 & 1.044 \\
\hline $\mathrm{F} / 224$ & 0.2774 & 2.431 & 2.999 & 5.299 \\
\hline $\mathrm{F} / 2 \mathrm{C}$ & 0.1659 & 0.2116 & 0.9683 & 1.865 \\
\hline
\end{tabular}

Table 4: Monte Carlo Simulated Concentration Estimates. All units are moles per liter. $2 \mathrm{C}$ waste does not contain any $\mathrm{La}$ or $\mathrm{Mn}$, and 224 waste contains no $\mathrm{Si}_{3}$ in Agnew's historical model.

As noted previously, these results have value in and of themselves. They provide an assessment of the uncertainty inherent in the historical estimates due to imprecise knowledge of the relevant parameters. They describe a reasonable range of values that could be observed for the estimates. However, the usefulness of these estimates is contingent on the underlying model itself being approximately correct; it is also contingent on the choice of parameters to be varied in the Monte Carlo study. Future work should investigate extensions beyond those assumptions. 


\section{B The Bayesian Analysis}

This section describes the prior distributions, the likelihood function for the analytical data, and the combination of those two components to form the posterior distribution.

\section{B.1 Analytical Data and the Likelihood Function}

As in the various tank characterization reports (TCRs), a random effects analysis of variance (ANOVA) model is used for the analytical data. This data is obtained by laboratory analyses of core segments, and the variability in the data is assumed to result from the following sources:

1. Variability between the cores

2. Variability between replicate samples from the same core

3. Other sources of variability, including laboratory error

In mathematical terms, the model can be expressed as

$$
y_{i j k}=\mu+\alpha_{i}+\beta_{i j}+\epsilon_{i j k}
$$

where $y_{i j k}$ is the observed concentration in the $k$ th replicate analysis of the $j$ th composite drawn from the $i$ th core. The constant $\mu$ is the overall mean value, while $\alpha, \beta$ and $\epsilon$ are, respectively, random fluctuations due to spatial heterogeneity, incomplete mixing of composites, and other sources of error. The following distributional assumptions are made:

$$
\begin{aligned}
\alpha_{i} & \sim N\left(0, \sigma_{a}^{2}\right) \\
\beta_{i j} & \sim N\left(0, \sigma_{b}^{2}\right) \\
\epsilon_{i j k} & \sim N\left(0, \sigma_{e}^{2}\right)
\end{aligned}
$$

It is also assumed the three error terms are independent of one another.

The likelihood function derived from this model has the following form (assuming that there are I cores, $\mathrm{J}$ composites, and $\mathrm{K}$ replications):

$$
\begin{aligned}
l\left(\mu, \sigma_{e}^{2}, \sigma_{b}^{2}, \sigma_{a}^{2} ; y\right) \propto & \left(\sigma_{e}^{2}\right)^{-v_{1} / 2}\left(\sigma_{e}^{2}+K \sigma_{b}^{2}\right)^{-v_{2} / 2}\left(\sigma_{e}^{2}+K \sigma_{b}^{2}+J K \sigma_{a}^{2}\right)^{-\left(v_{3}+1\right) / 2} \\
& \times \exp \left[-\frac{1}{2}\left(\frac{I J K(\bar{y} \ldots-\mu)^{2}}{\sigma_{e}^{2}+K \sigma_{b}^{2}+J K \sigma_{a}^{2}}+\frac{v_{3} m_{3}}{\sigma_{e b a}^{2}}+\frac{v_{2} m_{2}}{\sigma_{e}^{2}+K \sigma_{b}^{2}}+\frac{v_{1} m_{1}}{\sigma_{e}^{2}}\right)\right]
\end{aligned}
$$

where

$$
\begin{aligned}
v_{1} & =I J(K-1) \\
v_{2} & =I(J-1) \\
v_{3} & =I-1 \\
m_{1} & =\sum_{i, j, k}\left(y_{i j k}-\bar{y}_{i j .}\right)^{2} / v_{1}
\end{aligned}
$$




$$
\begin{aligned}
& m_{2}=K \sum_{i, j}\left(\bar{y}_{i j .}-\bar{y}_{i . .}\right)^{2} / v_{2} \\
& m_{3}=J K \sum_{i}\left(\bar{y}_{i . .}-\bar{y}_{. . .}\right)^{2} / v_{3}
\end{aligned}
$$

\section{B.2 Prior Distributions}

The distributions estimated for the analyte concentrations, described in Appendix A, are used as the prior distributions for the $\mu$ values. It is also necessary to place prior distributions on all of the variance parameters.

\section{B.2.1 Prior on $\mu$}

The pdf on the historical model prediction of the the analyte concentration is used as the prior distribution for $\mu$, and will be written $\pi(\mu)$. One thousand realizations were drawn from this distribution in the Monte Carlo study. One way to estimate the pdf is to place a point mass of $1 / 1000$ at each simulated value. However, for purposes of graphical display, a smoothed density estimate is preferred. A variable-span kernel density estimate for $\pi(\mu)$ was found, using a Gaussian kernel.

This density estimate is, in practice, evaluated on a fine grid of values of $\mu$. However, should it be necessary, an estimate of the pdf $\pi(\mu)$ can be obtained for any value $\mu$.

\section{B.2.2 Priors on the Variance Parameters}

Data on ICP laboratory analyses of reference samples was used to calibrate the prior on the error variance, $\pi\left(\sigma_{e}\right)$. These data are reproduced in Appendix C. The other priors were chosen by making assumptions about the relative scale of the various errors.

The laboratory accuracies were reported in terms of the deviations in the percent recovery. That is, if $a$ is the known concentration of a reference sample and $b$ is the observed value, then the value reported was the estimated standard deviation of

$$
100 b / a
$$

If we write $s$ as this percentage standard deviation, then the variance of the error on the concentration scale is

$$
a^{2} s^{2} / 100^{2}
$$

These 31 quantities will be referred to as the laboratory standard variances (LSVs).

The sample of LSVs is treated as a sample from a probability distribution. The most convenient distribution is the inverse gamma distribution. This distribution can be fit by the method of moments. The inverse gamma distribution is

$$
f(\nu)=\frac{\lambda^{r} \nu^{-(r+1)}}{\Gamma(r)} \exp (-\lambda / \nu)
$$


its mean and variance are given by

$$
\mathrm{E}(\nu)=\frac{\lambda}{r-1} ; \quad \operatorname{Var}(\nu)=\frac{\lambda^{2}}{(r-1)^{2}(r-2)}
$$

If the mean and variance of the LSVs are observed to be $\bar{x}$ and $s^{2}$ respectively, then the method of moments estimates for $r$ and $\lambda$ are

$$
\begin{aligned}
& r=2+\bar{x}^{2} / s^{2} \\
& \lambda=(r-1) \bar{x} .
\end{aligned}
$$

It is assumed that the prior mean of $\sigma_{e}^{2}$ is 100 times the mean of the LSVs; this reflects the fact that laboratory standard measurements are made under ideal circumstances, and the reference solution contains no extraneous materials. It is assumed the prior variance of $\sigma_{e}^{2}$ is 15,000 times the variance of the LSVs. This inflation factor is arrived at in the following manner. If the mean is inflated by a factor of 100 , the variance is inflated by a factor of 10,000. This inflation was then increased by fifty percent to account for the fact that we really have very little idea, a priori, what the value of $\sigma_{e}^{2}$ should be. In this way, we avoid overstating the certainty of our knowledge about $\sigma_{e}^{2}$, which could happen by assuming that the LSVs are entirely representative of the core sample variances.

It is further assumed the expected value of $\sigma_{b}^{2}$, the variability between composites, is 20 times that of $\sigma_{e}^{2}$, with a variance of 400 times that of $\sigma_{e}^{2}$. This adjustment is to reflect the a priori expectation that variability due to the difficulty in mixing the composites will be much greater than the laboratory variability.

Finally, it is assumed that $\sigma_{a}^{2}$, the variability between cores, has an expected value which is 100 times that of $\sigma_{b}^{2}$, with a variance which is 10,000 times as great. This adjustment is to reflect the fact that the greatest source of variability is expected to be the spatial variability.

For computational convenience, the actual priors used are priors on the following quantities :

$$
\begin{array}{r}
\sigma_{e}^{2} \\
\sigma_{e}^{2}+2 \sigma_{b}^{2} \\
\sigma_{e}^{2}+2 \sigma_{b}^{2}+4 \sigma_{a}^{2}
\end{array}
$$

The features of the individual $\sigma^{2}$ terms described are used to determine the priors on the variance parameters. The values of those parameters can be found in Appendix C.

\section{B.3 Combination of Prior and Likelihood}

Because the quantity of greatest interest is the concentration of an analyte, $\mu$, the variance parameters can be integrated out in the following manner,

$$
l(\mu ; y)=\int_{\sigma_{e}^{2}, \sigma_{a}^{2}, \sigma_{b}^{2}} l\left(\mu, \sigma_{c}^{2}, \sigma_{a}^{2}, \sigma_{b}^{2} ; y\right) \pi\left(\sigma_{c}^{2}, \sigma_{a}^{2}, \sigma_{b}^{2}\right) d \sigma_{e}^{2} d \sigma_{a}^{2} d \sigma_{b}^{2}
$$


where $l\left(\mu, \sigma_{e}^{2}, \sigma_{a}^{2}, \sigma_{b}^{2} ; y\right)$ is as given in expression (2). After this function is found, the posterior distribution for $\mu$ is given by

$$
f(\mu \mid y)=\frac{l(\mu ; y) \pi(\mu)}{\int_{\mu} l(\mu ; y) \pi(\mu) d \mu} \propto l(\mu ; y) \pi(\mu)
$$

The intent is to simplify the problem so that only one parameter, $\mu$, need be considered. The technique described here allows that, while taking into full account the effect of the uncertainty in the parameters that are being eliminated. Using point estimates of the variance parameters has no formal justification in this context. With the small amount of data available, point estimates of those variance parameters can be quite unstable. The empirical Bayes approach, which finds such point estimates and then assumes they are correct, would greatly underestimate the uncertainty of a Bayesian estimate of $\mu$.

A relevant calculation is worked out in section A5.2 of Box and Tiao [4]:

$$
\begin{aligned}
\int_{x_{3}>x_{2}>x_{1}>0} & x_{1}^{-\left(p_{1}+1\right)}\left(x_{1}+c_{1} x_{2}\right)^{-\left(p_{2}+1\right)}\left(x_{1}+c_{1} x_{2}+c_{2} x_{3}\right)^{-\left(p_{3}+1\right)} \\
& \times \exp \left[-\left(\frac{a_{1}}{x_{1}}+\frac{a_{2}}{x_{2}}+\frac{a_{3}}{x_{3}}\right)\right] d x_{1} d x_{2} d x_{3} \\
& =\left(c_{1} c_{2}\right)^{-1}\left(\prod_{i=1}^{3} \Gamma\left(p_{i}\right) a_{i}^{-p_{i}}\right) \operatorname{Pr}\left(\frac{\chi_{2 p_{1}}^{2}}{\chi_{2 p_{2}}^{2}}>\frac{a_{1}}{a_{2}}, \frac{\chi_{2 p_{2}}^{2}}{\chi_{2 p_{3}}^{2}}>\frac{a_{2}}{a_{3}}\right)
\end{aligned}
$$

Because the joint prior on the variances is as follows:

$$
\begin{aligned}
\pi\left(\sigma_{e}^{2}, \sigma_{b}^{2}, \sigma_{a}^{2}\right) \propto & \left(\sigma_{e}^{2}\right)^{-r_{e}-1}\left(\sigma_{e}^{2}+K \sigma_{b}^{2}\right)^{-\tau_{e b}-1}\left(\sigma_{e}^{2}+K \sigma_{b}^{2}+J K \sigma_{a}^{2}\right)^{-r_{e b a}-1} \\
& \exp \left(\frac{-\lambda_{e}}{\sigma_{e}^{2}}-\frac{\lambda_{e b}}{\sigma_{e}^{2}+K \sigma_{b}^{2}}-\frac{\lambda_{e b a}}{\sigma_{e}^{2}+K \sigma_{b}^{2}+J K \sigma_{a}^{2}}\right)
\end{aligned}
$$

the product of the likelihood and the prior on the variances is

$$
\begin{aligned}
l\left(\mu, \sigma_{e}^{2}, \sigma_{b}^{2}, \sigma_{a}^{2} ; y\right) \pi\left(\sigma_{e}^{2}, \sigma_{b}^{2}, \sigma_{a}^{2}\right) \propto & \\
& \left(\sigma_{e}^{2}\right)^{-v_{1} / 2-r_{e}-1}\left(\sigma_{e}^{2}+K \sigma_{b}^{2}\right)^{-v_{2} / 2-r_{e b}-1}\left(\sigma_{e}^{2}+K \sigma_{b}^{2}+J K \sigma_{a}^{2}\right)^{-v_{3} / 2-r_{e b a}-3 / 2} \\
& \exp \left[-\frac{v_{1} m_{1}+\lambda_{e}}{\sigma_{e}^{2}}-\frac{v_{2} m_{2}+\lambda_{e b}}{\sigma_{e}^{2}+K \sigma_{b}^{2}}-\frac{v_{3} m_{3}+I J K\left(\bar{y}_{\ldots}-\mu\right)^{2}+\lambda_{e b a}}{\sigma_{e}^{2}+K \sigma_{b}^{2}+J K \sigma_{a}^{2}}\right](6)
\end{aligned}
$$

The integral of expression (6) with respect to the variance parameters is found to be

$$
\left(c_{1} c_{2}\right)^{-1}\left(\prod_{i=1}^{3} \Gamma\left(p_{i}\right) a_{i}^{-p_{i}}\right) \operatorname{Pr}\left(\frac{\chi_{2 p_{1}}^{2}}{\chi_{2 p_{2}}^{2}}>\frac{a_{1}}{a_{2}}, \frac{\chi_{2 p_{2}}^{2}}{\chi_{2 p_{3}}^{2}}>\frac{a_{2}}{a_{3}}\right)
$$

where

$$
\begin{aligned}
& c_{1}=K \\
& c_{2}=J K
\end{aligned}
$$




$$
\begin{aligned}
& p_{1}=v_{1} / 2+r_{e} \\
& p_{2}=v_{2} / 2+r_{e b} \\
& p_{3}=v_{3} / 2+r_{e b a}+1 / 2 \\
& a_{1}=v_{1} m_{1}+\lambda_{e} \\
& a_{2}=v_{2} m_{2}+\lambda_{e b} \\
& a_{3}=v_{3} m_{3}+I J K\left(\bar{y}_{\ldots}-\mu\right)^{2}+\lambda_{e b a} .
\end{aligned}
$$

\section{B.4 Calculation of the Posterior of $\mu$}

This calculation is relatively straightforward. The expression (7) for the integrated likelihood is multiplied by the value of the prior for each value of $\mu$, as in (4). In practice, this amounts to evaluating the product $l(\mu ; y) \pi(\mu)$ for a grid of values of $\mu$ and normalizing the result so that it is a pdf.

This pdf is the posterior distribution of $\mu$ given $y$, and all subsequent inference can be based on it. For point estimates, the mean can be reported. The standard deviation of the distribution provides an uncertainty estimate, while interval estimates are given by percentiles of the posterior distribution.

\section{B.5 Updating the Historical Model Parameters}

This section describes methods for updating the historical model based upon the data. These methods have not been implemented.

We write $\phi$ for the set of parameters in the historical model which were varied in the Monte Carlo study. The concentration parameter $\mu$ is related to $\phi$ by some equation implicit in the historical model, which we will write

$$
\mu=g(\phi)
$$

The prior pdf for $\phi$ will be written as $\nu(\phi)$.

The likelihood of $\phi$ rather than $\mu$ can be found as follows:

$$
l_{\phi}(\phi ; y)=l(\mu=g(\phi) ; y)
$$

where $l(\mu ; y)$ is as derived in Section B.3.

Thus, the posterior distribution of $\phi$ is given by

$$
f(\phi \mid y) \propto l_{\phi}(\phi ; y) \nu(\phi)
$$

As with $\mu$, in practice this product is evaluated over a grid of $\phi$ values and then normalized so that it is a pdf.

It should be noted that this discussion is valid only for the analysis of one analyte; simultaneous consideration of more analytes that depend on the same input parameters will require a more sophisticated approach. 


\section{B.6 Predictions}

Here we consider the problem of making predictions about some unsampled tank, given the observed data.

The tanks analyzed in this preliminary study are, for purposes of the historical model, composed of only one waste type. Thus, the concentrations that are estimated for the tank also serve as estimates for the waste type. If another tank contains some mixture of these waste types (and no other types of waste), the posterior for each waste type can be weighted according to its proportion and summed to form a posterior for the unsampled, mixed waste type tank.

In mathematical terms, if $\mu_{n}$ is the concentration in the unsampled tank, and $\mu_{1}$ and $\mu_{2}$ are the concentrations of the same analyte for two different waste types, then

$$
\mu_{n}=p_{1} \mu_{1}+\left(1-p_{1}\right) \mu_{2}
$$

where $p_{1}$ is the proportion of the unsampled tank that is composed of the first type of waste.

The posterior mean and variance of $\mu_{n}$ can be easily calculated from the posterior moments :

$$
\begin{array}{r}
\mathrm{E}\left(\mu_{n} \mid y\right)=p_{1} \mathrm{E}\left(\mu_{1}\right)+\left(1-p_{1}\right) \mathrm{E}\left(\mu_{2}\right) \\
\operatorname{Var}\left(\mu_{n} \mid y\right)=p_{1}^{2} \operatorname{Var}\left(\mu_{1} \mid y\right)+p_{2}^{2} \operatorname{Var}\left(\mu_{2} \mid y\right)
\end{array}
$$

The variance expression assumes that the $\mu_{1}$ and $\mu_{2}$ are independent of one another a posteriori. In the specific case described here, this is true, because the posteriors for the two concentrations came from two different data sets. In general, however, this expression may not apply.

The posterior distribution of $\mu_{n}$ can be found by

$$
\left.f\left(\mu_{n} \mid y\right)=\int \mu_{1} f_{(} \mu_{1}, \mu_{2}=\left(\mu_{n}-p_{1} \mu_{1}\right) /\left(1-p_{1}\right) \mid y\right) d \mu_{1}
$$

Alternately, if the approach from the previous section is applied, the result is a posterior distribution on $\phi$, the set of parameters from the historical model. A sample can be drawn from this posterior pdf, much as was done in the Monte Carlo study described in this paper, and put through the historical model. Given the previous data, the resulting set of estimates would serve as a sample from the posterior distribution of the new tank. If data then became available for the (previously unsampled) tank, it could be used in a Bayesian analysis to form a posterior distribution in the same manner as the data considered in this paper. 


\section{Parameter Values Used in Prior Distributions}

The volume percent solid parameter for $2 \mathrm{C}$ waste is assumed, a priori, to have the following distribution. If $\theta$ is $\operatorname{beta}(7.102804,11.8972)$, the volume percent solid has the same distribution as.

$$
10.7 \theta+2.8
$$

These parameters give a distribution on the range $(2.8,13.5)$ with a mean value of 6.8 .

The volume percent solid parameter for 224 waste is assumed, a priori, to have the following distribution. If $\theta$ is beta $(1.028037,8.971963)$, the volume percent solid has the same distribution as

$$
10.7 \theta+2.8
$$

These parameters give a distribution on the range $(2.8,13.5)$ with a mean value of 3.9 .

The variance of a beta distribution increases as the sum of the beta parameters decreases. The priors for $2 \mathrm{C}$ versus 224 have the desired property, namely, that 224 has a considerably higher variability.

The priors for the variances on the ICP measurements were derived from the laboratory standards data shown in Table 5, reproduced from [5]. The priors that were derived for the variance parameters are given in Table 6 . The parameters given are for the inverse gamma distribution,

$$
f(\nu)==\frac{\lambda^{r} x^{-(r+1)}}{\Gamma(r)} \exp (-\lambda / x) .
$$




\begin{tabular}{|c|r|r|r|}
\hline Analyte & Avg. \% Recov. & Rel. Std. Dev. & Standard Conc. - ppm \\
\hline $\mathrm{Al}$ & 96.7 & 4.9 & 4.978 \\
\hline $\mathrm{Sb}$ & 100.2 & 3.0 & 4.946 \\
\hline $\mathrm{As}$ & 99.6 & 2.5 & 5.008 \\
\hline $\mathrm{Ba}$ & 96.9 & 2.6 & 5.089 \\
\hline $\mathrm{Be}$ & 102.3 & 3.2 & 5.269 \\
\hline $\mathrm{Cd}$ & 98.5 & 3.5 & 5.252 \\
\hline $\mathrm{Ca}$ & 96.5 & 3.5 & 5.023 \\
\hline $\mathrm{Cr}$ & 100.3 & 3.0 & 5.289 \\
\hline $\mathrm{Co}$ & 98.6 & 2.0 & 5.266 \\
\hline $\mathrm{Cu}$ & 96.5 & 1.8 & 5.132 \\
\hline $\mathrm{Fe}$ & 98.5 & 2.7 & 5.156 \\
\hline $\mathrm{Pb}$ & 98.3 & 4.7 & 5.149 \\
\hline $\mathrm{Mg}$ & 98.1 & 3.7 & 4.902 \\
\hline $\mathrm{Mn}$ & 95.0 & 2.8 & 5.153 \\
\hline $\mathrm{Ni}$ & 98.9 & 2.7 & 5.186 \\
\hline $\mathrm{K}$ & 99.3 & 2.9 & 5.232 \\
\hline $\mathrm{Se}$ & 100.1 & 2.9 & 5.074 \\
\hline $\mathrm{Ag}$ & 99.1 & 4.9 & 5.101 \\
\hline $\mathrm{Na}$ & 96.1 & 3.5 & 5.097 \\
\hline $\mathrm{V}$ & 98.9 & 2.3 & 5.159 \\
\hline $\mathrm{Zn}$ & 99.0 & 1.4 & 5.232 \\
\hline $\mathrm{Bi}$ & 100.1 & 2.4 & 5.014 \\
\hline $\mathrm{B}$ & 95.8 & 4.7 & 5.018 \\
\hline $\mathrm{La}$ & 103.2 & 3.0 & 5.101 \\
\hline $\mathrm{P}$ & 96.9 & 4.2 & 5.098 \\
\hline $\mathrm{Si}$ & 94.4 & 5.1 & 4.712 \\
\hline $\mathrm{Sr}$ & 96.3 & 2.2 & 5.052 \\
\hline $\mathrm{S}$ & 100.1 & 3.2 & 5.204 \\
\hline $\mathrm{Ti}$ & 98.1 & 2.2 & 4.980 \\
\hline $\mathrm{Zr}$ & 101.4 & 3.8 & 5.077 \\
\hline $\mathrm{Ce}$ & 103.9 & 1.3 & 5.170 \\
\hline & & & \\
\hline
\end{tabular}

Table 5: Precisions of estimates of laboratory standards. 


\begin{tabular}{|c|c|c|}
\hline Quantity & $r$ & $\lambda$ \\
\hline$\sigma_{e}^{2}$ & 3.710605 & 7.385209 \\
\hline $2 \sigma_{b}^{2}+\sigma_{e}^{2}$ & 3.796082 & 312.3419 \\
\hline $4 \sigma_{a}^{2}+2 \sigma_{b}^{2}+\sigma_{e}^{2}$ & 3.72814 & 59768.64 \\
\hline
\end{tabular}

Table 6: Prior parameters for variance quantities. 


\section{Distribution}

No. of

Copies

OFFSITE

2 DOE/Office of Scientific and Technical Information

\section{ONSITE}

3 Westinghouse Hanford Company

T. M. Brown

R2-12

S. J. Eberlein

B. C. Simpson

R2-12

R2-12
No. of
Copies

10 Pacific Northwest Laboratory

G. Chen

K5-12

A. F. Liebetrau

K5-12

A. F. Noonan

K9-81

K. M. Remund

K5-12

F. M. Ryan

Technical Report Files (5)

Distr. 1 